\title{
Enhancing and inhibiting star formation: high-resolution simulation studies of the impact of cold accretion, mergers and feedback on individual massive galaxies
}

\author{
Leila C. Powell ${ }^{1}$, Frederic Bournaud ${ }^{2}$, Damien Chapon ${ }^{2}$, \\ Julien Devriendt ${ }^{3}$, Volker Gaibler ${ }^{4}$, Sadegh Khochfar ${ }^{1}$, \\ Adrianne Slyz ${ }^{3}$ and Romain Teyssier ${ }^{2}$ \\ ${ }^{1}$ Max Planck Institute for extraterrestrial Physics, PO Box 1312, Giessenbachstr., \\ 85741 Garching, Germany \\ email: lpowell@mpe.mpg.de \\ ${ }^{2}$ Service d'astrophysique, CEA, Orme des Merisiers, Gif-sur-Yvette Cedex, France \\ ${ }^{3}$ Oxford astrophysics, Denys Wilkinson Building, Keble Road, OX1 3RH \\ ${ }^{4}$ Universität Heidelberg, Zentrum für Astronomie, Institut für Theoretische Astrophysik, \\ Albert-Ueberle-Str. 2, 69120 Heidelberg, Germany
}

\begin{abstract}
The quest for a better understanding of the evolution of massive galaxies can be broadly summarised with 2 questions: how did they build up their large (stellar) masses and what eventually quenched their star formation (SF)? To tackle these questions, we use highresolution RAMSES simulations (Teyssier 2002) to study several aspects of the detailed interplay between accretion (mergers and cold flows), SF and feedback in individual galaxies. We examine $\mathrm{SF}$ in major mergers; a process crucial to stellar mass assembly. We explore whether the mergerinduced, clustered SF is as important a mechanism in average mergers, as it is in extreme systems like the Antennae. We find that interaction-induced turbulence drives up the velocity dispersion, and that there is a correlated rise in SFR in all our simulated mergers as the density pdf evolves to have an excess of very dense gas. Next, we introduce a new study into whether mechanical jet feedback can impact upon the ability of hot gas haloes to provide a supply of fuel for SF during mergers and in their remnants. Finally, we briefly review our recent study, in which we examine the effect of supernova (SN) feedback on galaxies accreting via the previously overlooked coldmode, by resimulating a stream-fed galaxy at $z \sim 9$. A far-reaching galactic wind results yet it cannot suppress the cold, filamentary accretion or eject significant mass in order to reduce the SFR, suggesting that SN feedback may not be as effective as is often assumed.
\end{abstract}

Keywords. methods: numerical, galaxies: evolution, galaxies: star clusters, galaxies: interactions, galaxies: high-redshift, galaxies: starburst, galaxies: jets, stars: supernovae: general

\section{Clumpy star formation induced by mergers}

It has been widely demonstrated with previous generations of merger simulations, that merging galaxies undergo a nuclear starburst. In this picture, gas is driven to the centre by tidal torques, where it is compressed and turns into stars at a very high rate (Barnes \& Hernquist 1991). This was compatible with observations of ULIRGs which exhibit centrally concentrated star formation. However, there is now significant observational evidence of the existence of merging galaxies whose star formation is extended and clustered (e.g. the Antennae), as well as attempts by simulators to model these specific systems (Teyssier et al. 2010). We undertake a more general study of clustered star formation by performing 5 idealised simulations of equal-mass mergers (with $\sim 5 \mathrm{pc}$ 

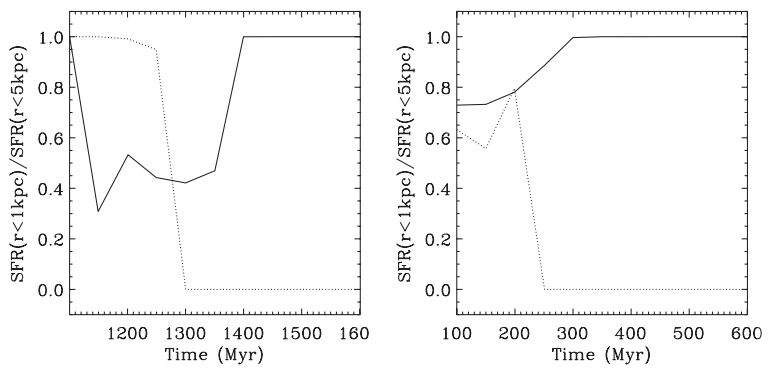

Figure 1. The ratio of the SFR within a $1 \mathrm{kpc}$ radius to that within $5 \mathrm{kpc}$ radius for galaxy 1 (solid line) and galaxy 2 (dotted line - when this goes to zero, galaxy 2 has merged with galaxy 1). Left: Merger m5. Right: Merger m3.
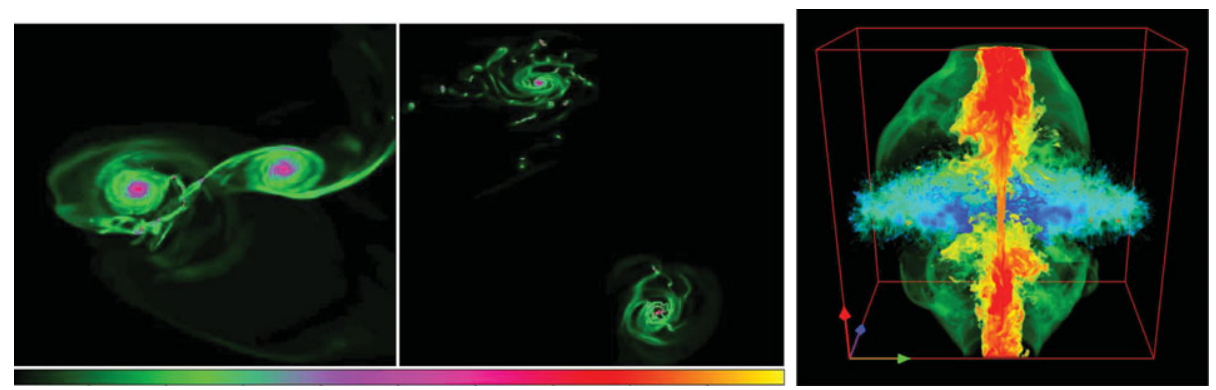

Figure 2. Left(2 panels): Gas density map to illustrate the increased clumpiness of the gas as merger m5 progresses. There is a 100Myr time difference between the left and right panels - initially both discs are relatively smooth, but one becomes noticeably more clumpy. Right: Image of jet interacting with cold gas disc and a hot ambient medium. See Gaibler et al. (2012) for details of the jet modeling.

resolution) with orbital parameters that can be considered 'average' for a LCDM cosmology. The full results of this study will be presented in Powell et al. (2012, in prep).

\subsection{Extended, clustered star formation}

In Fig. 1 we show the ration of the SFR within $1 \mathrm{kpc}$ to that within $5 \mathrm{kpc}$ for galaxy 1 (solid line) and galaxy 2 (dotted line) in mergers m5 (left-hand panel) and m3 (righthand panel). When this quantity is 1 , all SF is within the central $1 \mathrm{kpc}$ and so it could be considered to be a 'nuclear' starburst. When the quantity is lower it indicates some of the $\mathrm{SF}$ is occurring beyond $1 \mathrm{kpc}$ and so could be considered 'extended'. Here we see two extreme examples. Merger $\mathrm{m} 3$ has very concentrated $\mathrm{SF}$, whereas merger $\mathrm{m} 5$ has a period of more extended star formation where the ratio reaches values of $\sim 0.5$, in between two phases where the SF is centrally concentrated. The appearance of new/larger clumps in merger $\mathrm{m} 5$ is illustrated in the density maps in Fig. 2. The SF of all the mergers tends towards being centrally concentrated as the galaxies approach coalescence. Although it seems that stars formed in the nucleus dominate the SFR at the very peak of the starburst, extended/clumpy star formation still occurs to a greater or lesser extent in most of the mergers in our sample. This process could lead to significant phases of enhanced SF before the main starburst, alter the stellar distribution in massive ellipticals and even have implications for the origin of globular clusters.

\subsection{How does the merger affect the gas properties?}

In order to try to understand the mechanisms responsible for the merger-induced clumpy $\mathrm{SF}$ we measure the evolution of some of the gas properties. Fig. 3 (left-hand panel) shows the time evolution of the $1 \mathrm{D}$ velocity dispersion (solid line) measured in 100pc cells and averaged over a cube of side-length $30 \mathrm{kpc}$, centred on one of the galaxies in merger $\mathrm{m} 4$. The dotted line shows the global SFR at the same times. It is interesting that the increase 

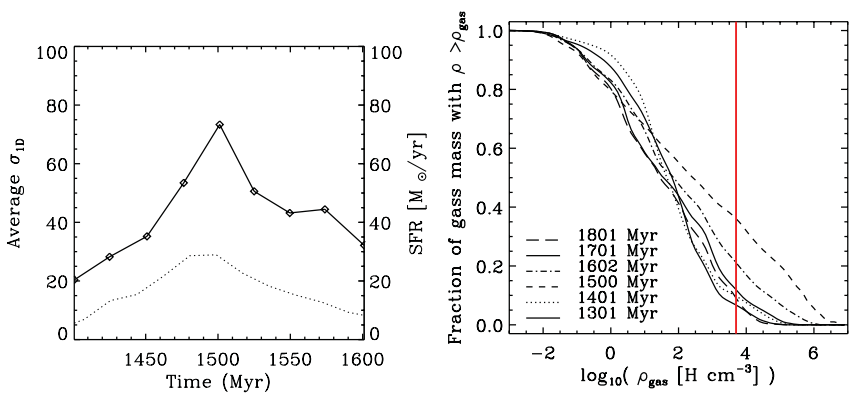

Figure 3. Left: The average 1D velocity dispersion within a $15 \mathrm{kpc}$ radius (solid line) and the global SFR (dotted line) for one of the mergers (m4). Right The cumulative gas density pdf within a radius of $15 \mathrm{kpc}$, also for merger $\mathrm{m} 4$. The vertical line shows the threshold for star formation.

in velocity dispersion is correlated with the increase in SFR and we note that this result is seen for all the mergers in our sample. There are two main possible sources of turbulence that could drive up the velocity dispersion: supernova feedback (which is included in the simulations) or the interaction itself. We rerun merger $\mathrm{m} 4$ for the duration of the starburst with supernova feedback switched off and find that the velocity dispersion curve remains the same, suggesting the interaction is driving increased turbulence.

Fig. 3 (right-hand panel) shows the time evolution of the gas density pdf for merger $\mathrm{m} 4$. It is clear that there is significant evolution of the pdf as the merger progresses (and as the velocity dispersion goes up, as just demonstrated). A large excess of very dense gas is created and this increases the amount of gas eligible for SF (the vertical line marks the SF threshold in the simulations). This trend is observed for all mergers.

\subsection{Revisiting the Kennicutt-Schmidt Relation}

There is ongoing debate about whether quiescent and starbursting galaxies form one or more sequences on the Kennicutt-Schmidt (KS) plot. Daddi et al. (2010) recently showed it was possible to fit two separate sequences and Teyssier et al. (2010) suggested that resolving clumpy star formation could be the key to reproducing/explaining this with simulations. Fig. 4 shows examples of the evolution of a merging galaxy on the KS plot. We do not see strong evidence for a clear bimodality, but rather some scatter in between the two relations. We note that merger m5 (left-hand panel) occurs after a long time has elapsed, which is why it starts below the disc sequence (the pre-merger disc has evolved away from the disc sequence).
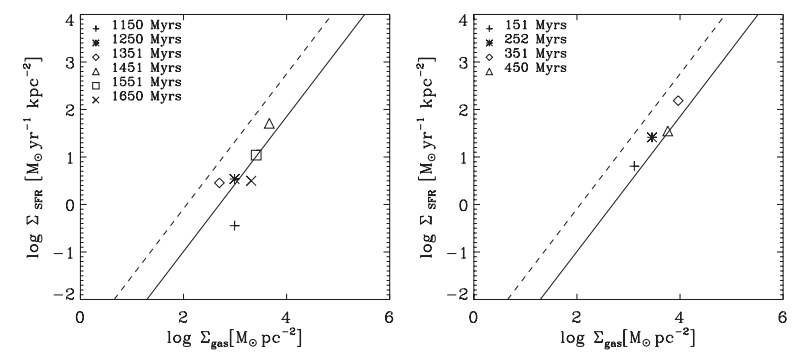

Figure 4. The time evolution of one galaxy from a merger on the Kennicutt-Schmidt plot. Fits from Daddi et al. (2010) for quiescent (solid line) and starbursting (dotted line) galaxies are overplotted. Left: Merger m5 a 'slow' merger. Right Merger m3, a 'rapid' merger.

\section{Star formation fueled by hot gas haloes in mergers}

Idealised merger simulations have been typically performed without hot gas haloes (including those discussed above). Recently, Moster et al. (2011) included this component and found it affects the remnant properties as it acted as a reservoir of fuel for later SF. However, the impact of strong feedback, such as that from an active galactic nuclei has not yet been taken into account. Jets could potentially keep the hot halo hot, preventing 
it from being a fuel supply. We are currently testing simulations which combine idealised merger simulations including hot gas haloes with the jet module presented in Gaibler et al. (2012) (see Fig. 2, right-hand panel), in order to address this issue.

\section{Star formation fueled by cold flows}

In addition to mergers, cold flows are another important source of fuel for SF. This is particularly true for low mass galaxies (which can't support stable virial shocks, Birnboim \& Dekel 2003) and at high redshift (where the filaments are narrower and denser). In the hierarchical picture, however, this also has a knock-on effect for galaxies at lower redshifts and higher masses (Keres et al. 2005). We also observe supernova-driven winds at highredshift and we expect feedback from these winds to be particularly effective at reducing, or shutting down star formation in low-mass galaxies (Dekel \& Silk 1986). Essentially then, we expect both cold flows and galactic winds to be effective simultaneously.

We perform ultra-high resolution $(\sim 0.5 \mathrm{pc})$ cosmological resimulations of the $z \sim 9$ progenitor of a Milky Way-like galaxy to follow how its evolution is influenced by the combined effects of cold gas accretion via filaments and a supernova-driven galactic wind. This resolution allows us to directly model the Sedov blastwave phase of supernovae. Due to the expansion and overlapping of 'bubbles' formed by the blastwaves, a high-speed wind is driven to several times the galaxy's virial radius. By comparison with a simulation without supernovae, we establish that the wind has had no effect on the accretion rate via the filaments and does not eject significant mass, so it is not able to reduce the SFR. This suggests SN feedback is not as effective (in this regime) as previously thought. We refer the reader to Powell et al. (2011) for the full details and results.

\section{Conclusions}

We have highlighted aspects of galaxy evolution in which refinements to the classical pictures are now required because of more detailed information gleaned from observations and/or simulations: the addition of clumpy star formation to the nuclear starburst model; the inclusion in merger studies of not just hot gas haloes, but the feedback that may affect them; and the impact that filaments (often neglected) can have on the predicted efficiency of supernova feedback. We have demonstrated that hydrodynamical simulations are an invaluable tool to investigate all these refinements, but note that having high resolution is absolutely crucial, or these affects are simply not captured.

\section{References}

Barnes, J. E. \& Hernquist, L. E. 1991, ApJ, 370, L65

Birnboim, Y. \& Dekel, A. 2003, MNRAS, 345, 349

Daddi, E., Elbaz, D., Walter, F., Bournaud, F., Salmi, F., Carilli, C., Dannerbauer, H., Dickinson, M., Monaco, P., \& Riechers, D. 2010, ApJ (Letters), 714, L118

Dekel, A. \& Silk, J. 1986, ApJ, 303, 39

Kereŝ, D., Katz, N., Weinberg, D. H., \& Davé, R. 2003, MNRAS, 363, 2

Gaibler, V., Khochfar, S., Krause, M., \& Silk, J. 2012, MNRAS, 425, 438

Moster, B. P., Macciò, A. V., Somerville, R. S., Naab, T., \& Cox, T. J. 2011, MNRAS, 415, 3750

Powell, L. C., Slyz, A., \& Devriendt, J. 2011, MNRAS, 414, 3671

Teyssier, R. 2002, A\&A, 385, 337

Teyssier, R., Chapon, D., \& Bournaud, F. 2010, ApJ (Letters), 720, L149 